\title{
Pneumomediastinum as a complication of cocaine abuse
}

\author{
Authors: Catriona Macrae, ${ }^{A}$ Christopher Brown, ${ }^{B}$ Christine Aiken ${ }^{C}$ and Ravi Jamdar ${ }^{D}$
}

\section{A 26-year-old male presented with a 24-hour history of pleuritic chest pain following intranasal cocaine insufflation. He was a smoker, cannabis and alcohol user. Cardiovascular and respiratory examinations were unremarkable. \\ His admission blood tests were within normal limits. The admission electrocardiogram (ECG) showed sinus rhythm, with ST-segment elevation in an inferolateral distribution. This appeared to be an early repolarisation abnormality, with no evolving changes.}

His chest radiogram showed a double outline at the left heart border with subcutaneous gas collection over the left supraclavicular fossa but no evidence of pneumothorax. A computed tomography (CT) showed prominent mediastinum with gas tracking into the neck but no connection to the oesophagus or pneumothorax.

He was managed conservatively and a repeat chest radiogram after 48 hours showed improvement.

KEYWORDS: Chest pain, cocaine, pneumomediastinum

\section{Introduction}

Cocaine use is increasingly prevalent in the UK as a stimulant drug of abuse. It is used recreationally for its sympathomimetic effects on the body. Recent data suggests that powdered cocaine for insufflation is the second most commonly used drug among adults aged between 16 and 59 years old within the UK. A recent Home Office report estimates 875,000 potential users in the UK. ${ }^{1}$ Both occasional recreational and chronic cocaine abuse is associated with a variety of serious medical pathologies, and here we present an unusual case of chest pain associated with acute cocaine insufflation.

\section{Case presentation}

A 26-year-old male presented to the acute medicine unit in Glasgow Royal Infirmary with a 24-hour history of severe chest pain.

Authors: ${ }^{\text {A }}$ core trainee year-1 acute care common stem, Glasgow Royal Infirmary, Glasgow, UK; ${ }^{B}$ acute medicine specialist registrar, Glasgow Royal Infirmary, Glasgow, UK; ' acute medicine consultant, Glasgow Royal Infirmary, Glasgow, UK; ' acute medicine consultant, Glasgow Royal Infirmary, Glasgow, UK
The patient reported a 'tight' and 'band-like' pain across his chest, associated with deep inspiration, and radiating to the throat and posterior thorax. The pain was of sudden onset, beginning immediately after the intranasal insufflation of two lines of powdered cocaine. In addition, he reported that he had been smoking cannabis heavily in the preceding 48 hours. He was a heavy smoker, reporting daily cigarette use, and regularly used alcohol. He denied any past medical history and had no previous relevant contact with hospital services.

On initial assessment in the acute assessment unit (AAU) he had reassuring admission observations. His respiratory rate was 16 breaths per minute, oxygen saturations were $99 \%$ on room air, his heart rate was 55 beats per minute and a non-invasive blood pressure was recorded as $148 / 88 \mathrm{mmHg}$. Cardiovascular examination was unremarkable; he was warm and well perfused. His heart sounds were pure and his lung fields were unremarkable on auscultation.

Given his history of stimulant use, immediate concern was of a new cardiac event. His admission blood test revealed a highsensitivity troponin I of $2 \mathrm{ng} / \mathrm{L}$. His C-reactive protein (CRP) was checked, and found to be within the normal range at $3 \mathrm{mg} / \mathrm{L}$. His remaining serum examinations were unremarkable, with normal renal and liver function, and unremarkable full blood count.

His admission electrocardiogram (ECG) confirmed an underlying sinus rhythm at 62 beats per minute, with a widespread (but nondiagnostic) ST-segment elevation in an inferolateral distribution. This appeared to be an early repolarisation abnormality and there were no evolving changes on serial ECG. Conduction was satisfactory with no evidence of heart block (Fig 1).

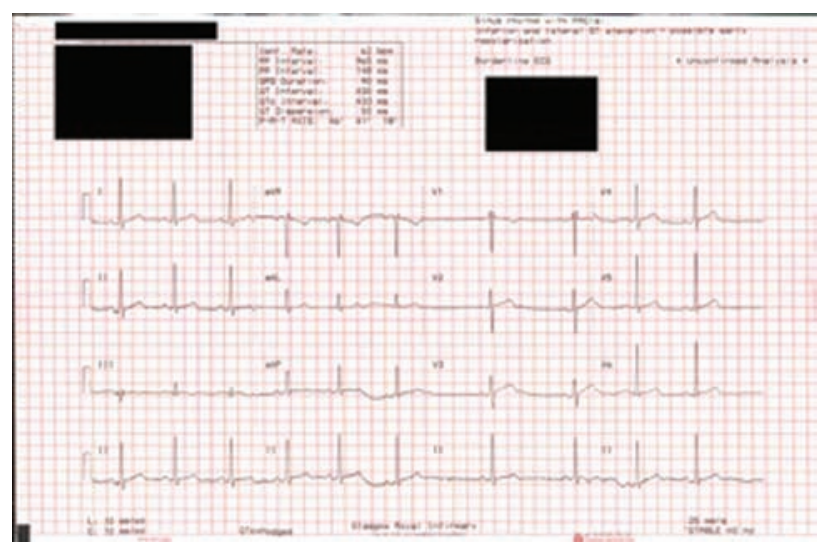

Fig 1. Admission electrocardiogram. 


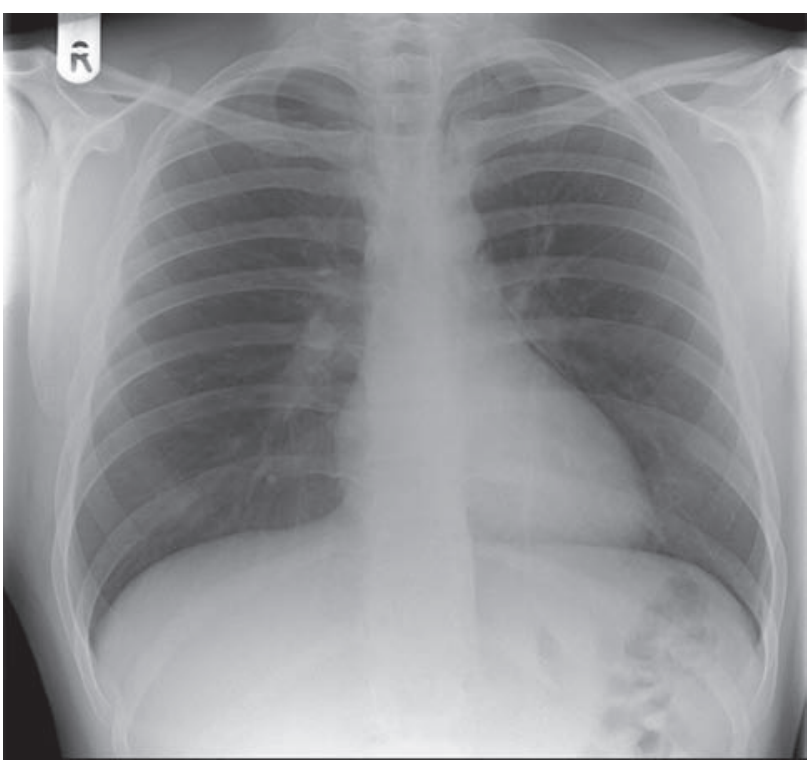

Fig 2. Chest radiogram demonstrating pneumomediastinum.

His admission chest radiogram showed a double outline at the left heart border, extending superiorly along the left mediastinum edge, with subcutaneous gas collection over the left supraclavicular fossa. There was no evidence of pneumothorax (Fig 2).

Given the concerning clinical history, and abnormal chest radiogram, the patient underwent emergency axial imaging to assess the mediastinum. Computed tomography (CT) of the thorax showed a prominent mediastinum with gas tracking up into the neck anteriorly. This CT did not show a mediastinal connection to the oesophagus or a pneumothorax (Fig 3, Fig 4).

\section{Diagnosis}

Pneumomediastinum secondary to intranasal insufflation of cocaine.

\section{Initial management}

The patient was admitted for observation and managed conservatively with input from the local respiratory medicine team.

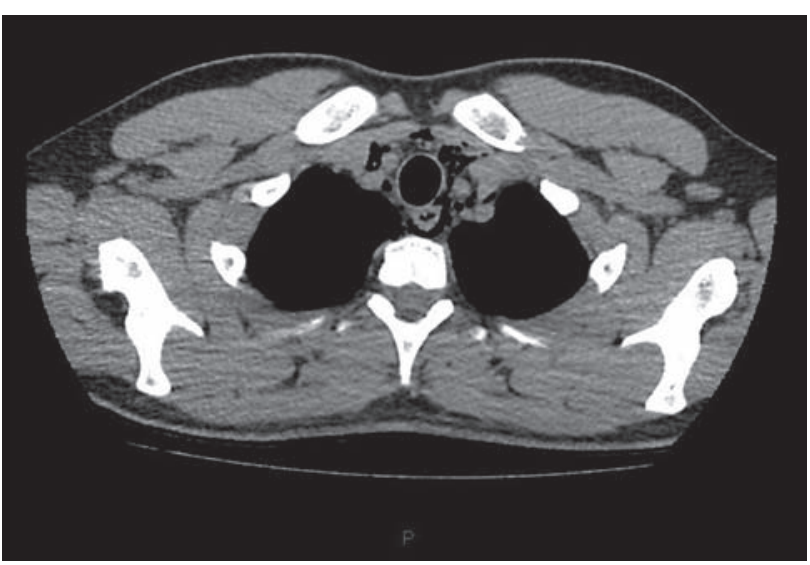

Fig 3. Computed tomography showing air in the mediastinum.

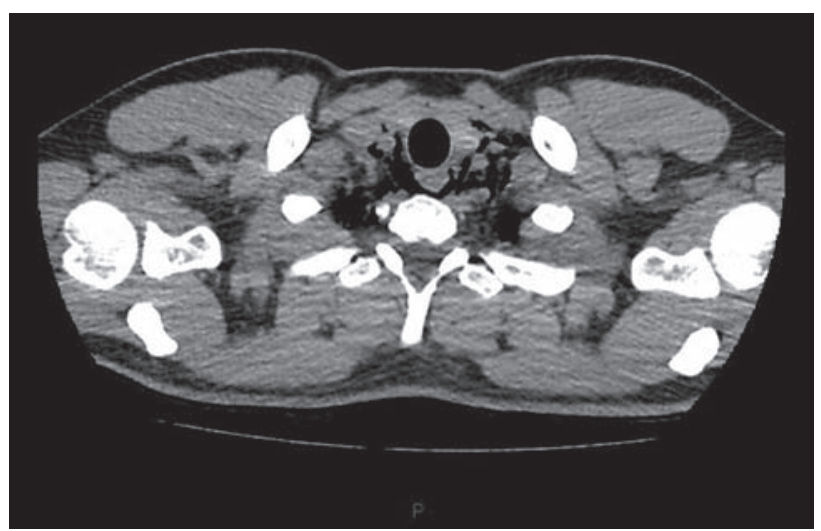

Fig 4. Computed tomography showing free air, tracking into the anterior neck.

He remained well and his symptoms improved significantly in the following 48 hours.

\section{Case progression and outcome}

Follow-up blood testing and ECG tracings were unchanged. CRP remained within the normal range throughout admission. A repeat chest radiogram 48 hours after admission showed improvement in the pneumomediastinum and he was discharged with safety advice. He was seen by the hospital addictions liaison team during this admission but he declined further support with his substance misuse or follow-up from the community addictions team.

\section{Discussion}

\section{Literature review}

Pneumomediastinum following smoking cocaine is well described $^{2,3}$ in cardiothoracic, emergency medicine and pulmonology literature, with multiple cases reported since the 1980 s. $^{3,4}$ It is now recognised that a similar presentation can occur following intranasal cocaine insufflation. ${ }^{5,6}$

A Brazilian multicentre study demonstrated multiple pulmonary changes on $\mathrm{CT}$ related to cocaine use, with the majority of the participants cocaine smokers, but also included patients with intranasal cocaine use. ${ }^{7}$ A Spanish case series looking at 46 patients with 'spontaneous' pneumomediastinum found that 26 of the patients had used cocaine prior to developing their symptoms. ${ }^{8}$ There are also a number of reports of pneumomediastinum where the method of cocaine ingestion is not documented. ${ }^{9,10}$

\section{Presentation}

The presenting features of pneumomediastinum secondary to intranasal cocaine use are most commonly chest pain, ${ }^{5,6}$ often described as pleuritic in nature, ${ }^{11-13}$ and/or dyspnoea. ${ }^{13-15}$ These features may present without tachycardia. ${ }^{14,16}$ One patient presented with asymptomatic surgical emphysema following cocaine insufflation without chest or retrosternal discomfort. ${ }^{17}$ Another indolent presentation occurred with sudden onset neck swelling, and progressive hoarseness following cocaine use. ${ }^{13}$ In keeping with UK data, there was a wide range of patient ages reported in the published cases series, ranging from 
15- to 61-years-old. ${ }^{11,18}$ Cocaine users often have a history of polysubstance abuse, with the concomitant use of alcohol, ${ }^{10,19}$ nitrous oxide, ${ }^{5}$ heroin, ${ }^{14}$ methylene-dioxy-methamphetamine $(\mathrm{MDMA})^{13}$ and cannabis ${ }^{13}$ all documented in cases of pneumomediastinum associated with cocaine.

In addition to pneumomediastinum, surgical emphysema ${ }^{15,16}$ and pneumorrhachis ${ }^{15,16,20}$ are also linked to nasal insufflation of cocaine. More serious manifestations can occur such Boerhaave's syndrome, with more extensive pneumomediastinum, pneumothorax, and fistulation with the distal oesophagus. ${ }^{18}$

\section{Investigation}

Pneumomediastinum is usually first identified on chest radiogram, $5,6,13$ with further axial imaging in the form of a CT carried out in many cases. ${ }^{6,13,16}$ Further imaging may be required to delineate the extent of the condition and to screen for the associated serious complications that can develop. Some clinicians advocate the use of barium swallows to rule out secondary causes of pneumomediastinum. ${ }^{17}$ Oesophageal microperforation was suspected in one case reviewed where no perforation was identified on barium swallow, but upper gastrointestinal endoscopy showed oesophagitis. ${ }^{6}$ The indications for and interpretation of barium swallow in this condition therefore remains unclear.

\section{Management}

Most patient histories we reviewed were admitted for observation ${ }^{5,12,17}$ and in some cases given prophylactic antibiotics. ${ }^{5,14}$ The majority were managed conservatively, ${ }^{6,11,12,15,19}$ with some patients observed in the intensive care setting for more intensive monitoring and nursing care. $^{5,11,16,18}$

Serious complications are reported in the literature, such as one case report that outlined a Boerhaave's syndrome and communicating pneumothorax. This patient developed septic shock and required emergency thoracotomy and emergency stent placement for management of pneumomediastinum associated with mediastinitis. ${ }^{18}$ Another patient with surgical emphysema required a surgical skin incision due to increasing neck swelling, to allow air drainage and relieve dyspnoea and tachypnoea. ${ }^{16}$

However, most cases are uncomplicated and managed conservatively with spontaneous resolution over $24-48$ hours, 5,11,14,15,17,19 with one patient observed for only 3 hours prior to hospital discharge. ${ }^{9}$

\section{Mechanism}

Different mechanisms for pneumomediastinum secondary to cocaine use have been postulated. Intranasal cocaine can corrode the nasal and respiratory muscosa. ${ }^{5}$ Boerhaave's syndrome is believed to be the result of a transmural oesophageal perforation secondary to these corrosive effects of cocaine on the oesophageal mucosa. ${ }^{18}$

Pneumomediastinum in cocaine users is widely ascribed to barotraumas during the insufflation process. Cocaine can cause bronchospasm, causing increased alveolar pressure ${ }^{14}$ that is further increased by forceful snorting. ${ }^{7}$ This effect is compounded by patients engaging in a Valsalva manoeuvre, reportedly carried out to increase the absorption and to potentiate the effect of cocaine. $^{8,14}$
The presumed mechanism for air escaping into the mediastinum and subcutaneous space is through alveolar rupture secondary to the creation of a significant pressure gradient ${ }^{11,17}$ over a short period of time. This leads to air passing into the interstitial tissues, which can then further track into the broncho-vascular sheath, through the fascial planes, into the deep neck spaces or even into the pleural cavity resulting in a pneumothorax. ${ }^{11,17}$

\section{Conclusion}

Pneumomediastinum is a recognised complication of intranasal cocaine insufflation. ${ }^{5,6,11,12,14,16,21}$ As in our case, most patients present with pleuritic chest pain, ${ }^{11-13}$ with or without tachycardia. $^{14,16}$ In the acute setting this can potentially lead to diagnostic uncertainty. There is often a high index of suspicion for cardiac disease in cases of chest pain associated with cocaine use due to the well-publicised risk of coronary artery vasospasm or arrhythmia; however, we postulate that pneumomediastinum is potentially considered less frequently. ${ }^{29}$ The diagnosis of pneumomediastinum is usually made on chest radiography, with more detailed imaging in the form of CT often employed. ${ }^{6,13,16}$

The majority of cases we reviewed in the literature were uncomplicated and suggest that these injuries resolve spontaneously with conservative management, 6,11,12,15,19 as described. However more serious complications such as pneumothorax, mediastinitis and sepsis may occur. ${ }^{18}$ There are different possible mechanisms behind this phenomenon but alveolar rupture secondary to increased alveolar pressure due to bronchoconstriction and the Valsalva manoeuvre is most commonly implicated. 8,14

Given the serious nature of potential complications, high prevalence of cocaine abuse and large number of patients presenting with chest pain, it is important for physicians working in the acute setting to be aware of this phenomenon and consider it during initial assessment and investigation to ensure appropriate management, in a timely manner.

\section{Key learning points}

> Pneumomediastinum is a complication of intranasal cocaine insufflation.

$>$ Most cases resolve spontaneously.

$>$ Complications such as pneumothorax, mediastinitis and sepsis can occur.

\section{References}

1 Home Office. Drug misuse: Findings from the 2017/18 crime survey for England and Wales. London: Home Office, 2018.

2 Lam PK, Wong TW, Lau CC. Pneumomediastinum after smoking cocaine: a case report and review of literature Hong Kong J Emerg Med 2011;18:26-30.

3 Morris JB, Shuck JM. Pneumomediastinum in a young male cocaine user. Ann Emerg Med 1985;14:194-6.

4 Fajardo L. Association of spontaneous pneumomediastinum with substance abuse West ] Med 1990;152:301-4.

5 Rampuri R, Ali Y, Verkerk M, Golding-Wood D. Unusual cause of pneumomediastinum BMJ Case Rep 2018;2018:bcr-2018224343

6 Chudasama K, Seenath M, Gourevitch D. Pneumomediastinum after cocaine use: an unusual aetiology. J Surg Case Rep 2010;2010:3.

7 Almeida RR, Zanetti G, Souza ASJr et al. Cocaine-induced pulmonary changes: HRCT findings. J Bras Pneumol 2015;41:323-30. 
8 Perna V, Vilà E, Guelbenzu J], Amat I. Pneumomediastinum: is this really a benign entity? When it can be considered as spontaneous? Our experience in 47 adult patients. Eur $]$ Cardiothorac Surg 2010;37:573-5.

9 Grau Jimenez MM, Sebastian GE, M Esparcia MG. Spontaneous pneumomediastinum and retropharyngeal emphysema: case report. Abstracts from the 8th Congress of the European Laryngological Society, 1-4 September 2010, Vienna, Austria. Eur Arch Otorhinolaryngol 2011;268:798.

10 Barbera Mir JA, Vallejo Galvete J, Velo Plaza M et al. Spontaneous pneumomediastinum after cocaine inhalation. Respiration 1986;50:230-2.

11 Conway EE. Spontaneous pneumomediastinum. Chest 1992;101:1743.

12 Stark P, Hartwick F, Dong Kim OK. Spontaneous pneumomediastinum as a consequence of cocaine abuse. Rofo 1988;148:323-4.

13 O'Dwyer D, Low TB, Neoftyou E, Mohammad A, McElvaney NG. Two case reports of pneumomediastinum. Ir J Med Sci 2007;176:239-41.

14 Ishikawa O, Jen H. Blown by blow: an usual etiology of pneumomediastinum. Am J Respir Crit Care Med 2018;197:A6635.

15 Kanu O, Teleb M, Agrawal H, Cashin LB. Cocaine-related subcutaneous emphysema, pneumorrhachis and pneumomediastinum: a rare clinical finding. BMJ Case Rep 2017;2017:bcr-2017-219851.
16 Atmaca Temrel T, Sener A, Icme F et al. Subcutaneous emphysema, pneumomediastinum, and pneumorrhachis after cocaine inhalation. Case Rep Emerg Med 2015;2015:134816.

17 Soares DS, Ferdman A, Alli R. Subcutaneous emphysema and pneumomediastinum following cocaine inhalation: a case report. J Med Case Rep 2015;9:195.

18 Van Leer-Greenberg B, Rapaka B, Mekaroonkamol P, Chawla S. Cocaine angina: A rare presentation of Boerhaave syndrome. Am J Gastroenterol 2017;112:S923-4.

19 Bond R, Duncan E, Nightingale A. Image of the month: Chest pain after cocaine use. Clin Med 2015;15:497-8.

20 Challita S, Daher M, Roche N et al. Pneumorachis after cocaine sniffing. Respir Med Case Reports 2014;12:10-12.

21 Kloss BT, Broton CE, Rodriguez E. Pneumomediastinum from nasal insufflation of cocaine. Int J Emerg Med 2010;3:435-7.

Address for correspondence: Dr Catriona Macrae, Acute Medical Department, Glasgow Royal Infirmary, Castle Street, Glasgow, G4 0SF, UK.

Email: catrionamacrae@nhs.net

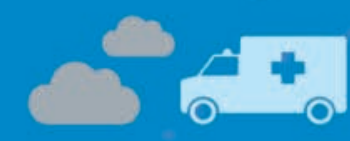

\section{Outpatients: the future - adding value through sustainability}

Published in 2018, this report from the Royal College of Physicians argues for

new models of outpatient care.

Download the report and supplementary case studies at www.rcplondon.ac.uk/outpatients-future-sustainability 